\title{
A case of manganese induced parkinsonism in hereditary haemorrhagic telangiectasia
}

\author{
K Yoshikawa, M Matsumoto, M Hamanaka, M Nakagawa
}

J Neurol Neurosurg Psychiatry 2003;74:1312-1314

A 44 year old right handed woman complained of difficulty in moving. She and her relatives had skin telangiectasia or recurrent epistaxis. On neurological examination, she had a mask-like facies and bradykinesia in both extremities. Laboratory examinations showed iron deficiency anaemia and mild liver dysfunction with raised serum manganese. On Tl weighted cranial magnetic resonance imaging there were hyperintense areas in the globus pallidus bilaterally, suggesting manganese deposition. Abdominal angiography confirmed multiple portalsystemic shunts in the liver, and a needle biopsy of the liver showed diffuse dilatation of the sinusoids with fatty change. Levodopa did not improve the bradykinesia. This appears to be a case of hereditary haemorrhagic telangiectasia with manganese induced parkinsonism, which may be a new type of neurological disorder in such patients.

$\mathrm{H}$ ereditary haemorrhagic telangiectasia, or Rendu-OslerWeber disease, was first reported by Rendu in 1896 as a systemic vascular disorder with idiopathic epistaxis and skin telangiectasia. ${ }^{1}$ It is now known to be caused by an abnormality of transforming growth factor $\beta$ binding proteins, and the patients show hereditary systemic vascular disorders in an autosomal dominant fashion with a high penetrance of $97 \%$ by the age of 50 years. ${ }^{2}$ Recurrent epistaxis or bleeding in the gastrointestinal tract during adolescence is the first symptom of the disease. In the fourth or fifth decade of life, chronic anaemia after recurrent haemorrhage, congestive heart failure caused by pulmonary arteriovenous fistulae, or liver dysfunction associated with portal-systemic shunting become evident, depending on the amount and localisation of the vascular abnormalities.

From the viewpoint of neurologists, the disease is interesting for its variety of complications in the central nervous system-paradoxical brain embolism or brain abscess in cases of pulmonary arteriovenous fistula (right to left shunt type, $61 \%)$; intracerebral haemorrhage or epilepsy in cases with arteriovenous malformations of the brain (parenchymal AVM type, $36 \%$ ); and hepatic coma with progression of portalsystemic shunt (porto-systemic shunt type, $3 \%$ ). ${ }^{3}$

In this study we present a patient with hereditary haemorrhagic telangiectasia with manganese induced parkinsonism. We propose that parkinsonism is a new neurological complication in this disease.

\section{CASE REPORT}

A 44 year old right handed woman consulted the neurological clinic because of difficulty in moving. This was especially marked during fine finger movements or in agility exercises. The symptom was insidious and had progressed over 12 months. She was a housewife and had never been exposed to manganese or its compounds. She did not smoke or drink. At the age of 40 years, duodenal ulcer and iron deficiency anaemia were diagnosed. There were no similar symptoms among her relatives. However, the patient, her father, and her younger brother all had skin or mucosal telangiectasia, and her son had experienced recurrent epistaxis, suggesting an autosomal dominant vascular disorder.

On examination, her vital signs were normal. Her conjunctivae were anaemic but not icteric. No Kayser-Fleischer rings was present in the corneas. There was no cyanosis and no pathological murmurs were heard in the chest. In the abdomen, neither liver nor spleen was palpable.

She was alert and oriented with normal results on digit span task, mini-mental state examination, and word fluency test. On neurological examination, no abnormality was found in the cranial nerves except for her face, which had a mask-like appearance, and her voice, which was very quiet. Muscle tone was generally increased with lead pipe rigidity and bradykinesia in all extremities. Motor power was $5 / 5$ in all muscles. Slight positioning tremor was present in both upper extremities. The deep tendon reflexes were normal and symmetrical. No pathological reflexes were elicited. Fingernose testing was normal and no dysdiadochokinesis was observed. Sensation was normal in vibration and pinprick tests. Posture on standing was normal, but arm swing while walking was reduced on both sides. No autonomic abnormality was found; in particular there was no orthostatic hypotension or constipation.

Laboratory studies showed iron deficiency anaemia with liver dysfunction: the red blood cell count was $382 \times 10^{4} / \mathrm{mm}^{3}$, haemoglobin was $9.2 \mathrm{mg} / \mathrm{dl}$, and the packed cell volume was $29.9 \%$. Serum iron was reduced to $1.6 \mu \mathrm{mol} / \mathrm{l}$. Serum alkaline phosphatase, $\gamma$-glutamyltransferase, and leucine aminopeptidase activities were increased to 504, 52, $243 \mathrm{U} / \mathrm{l}$, respectively. The levels of total protein, serum albumin, bilirubin, aspartate transaminase, alanine transaminase, glucose, electrolytes, urea nitrogen, and creatinine were all normal, and the ammonia level was within the normal limit $(28 \mu \mathrm{mol} / \mathrm{l})$. Amino acid analysis showed that phenylalanine, histidine, arginine, and tyrosine were raised, while valine, isoleucine, leucine, ornithine, and lysine were decreased, which led to a pronounced decrease in Fischer's ratio to 0.904 (normal, 2.6 to 4.2). Serum copper and caeruloplasmin were normal, but serum manganese was raised to $1.2 \mu \mathrm{mol} / \mathrm{l}$ (normal, 0.075 to $0.36 \mu \mathrm{mol} / \mathrm{l}$ ). The indocyanine green (ICG) plasma disappearance rate was decreased to $10.2 \%$ per minute (normal $>14.2 \%$ per minute). The decreased Fischer's ratio and ICG disappearance suggested either catabolic dysfunction of the liver or a portalsystemic shunt.

On cranial magnetic resonance imaging (MRI) there was no midbrain or tectal atrophy, putaminal atrophy with hypointense T2 weighted images, ischaemic change, or intracerebral haemorrhage, all of which have been found in cases of secondary parkinsonism syndromes such as progressive supranuclear palsy, Parkinson variant of multiple system atrophy, ${ }^{4}$ and vascular parkinsonism. ${ }^{5}$ On $\mathrm{Tl}$ weighted images, symmetrical ill defined hyperintense areas were found in the 


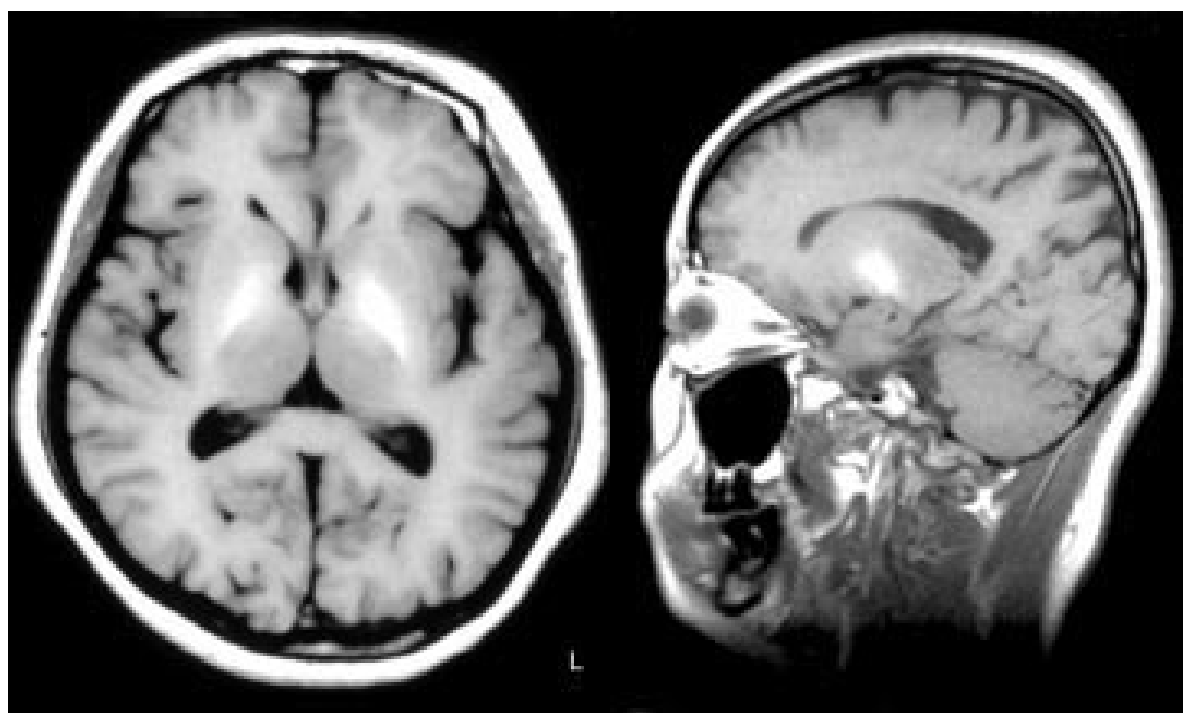

Figure $1 \mathrm{Tl}$ weighted magnetic resonance imaging of the brain in axial and sagittal sections. III defined high intensity areas are spread through the globus pallidus bilaterally. There is no macroscopic change compatible with progressive supranuclear palsy, the parkinsonian variant of multiple system atrophy, or vascular parkinsonism.

globus pallidus bilaterally, suggesting inappropriate deposition of metals such as iron, manganese, or calcium in the basal ganglia (fig 1). Magnetic resonance angiography was normal, with no evidence of arterial sclerosis or vascular malformations in the brain. Single photon emission computed tomography with $\left[{ }^{123} \mathrm{I}\right]-(\mathrm{IR})-2 \quad \beta$-carbomethoxy-3 $\quad \beta$ - $(4-$ iodophenyl)tropane was not possible, because this examination is not yet available in Japan. Electroencephalography showed a diffuse $\alpha$ pattern.

Ultrasound tomography of the abdomen revealed excess vasculature in the liver parenchyma. Abdominal angiography was carried out in a subsequent examination. Selective coeliac and superior mesenteric artery angiography showed dilated feeding arteries and early filling of the hepatic vein, with an abundant dense mottled hepatogram in the parenchyma, suggesting multiple submacroscopic portal-systemic shunts in the liver (fig 2, left panel). The patient underwent needle biopsy of the liver which showed diffuse dilatation of the sinusoids with fatty change in the liver tissue (fig 2, right panel).
A levodopa test with levodopa/DCI $100 \mathrm{mg}$ showed no significant change in scores on the unified Parkinson's disease rating scale, part $3^{6}$ (baseline, 19; 60 minutes after drug administration, 20). Administration of L-dopa in doses of up to $300 \mathrm{mg} /$ day for one month did not improve the clinical symptoms.

\section{DISCUSSION}

This patient had a familial history of an autosomal dominant vascular disorder, and abdominal angiography showed portalsystemic shunts in the liver, which is a typical finding in hereditary haemorrhagic telangiectasia, ${ }^{7}$ though we did not carry out genetic diagnosis for this condition. Removal of manganese from the portal circulation through the liver becomes incomplete in patients with portal-systemic shunts, and this leads to manganese deposition in the CNS. A raised serum manganese was confirmed in this patient, and cranial MRI showed hyperintensity in the globus pallidus bilaterally, suggesting manganese deposition in the brain. ${ }^{8}$ It is known
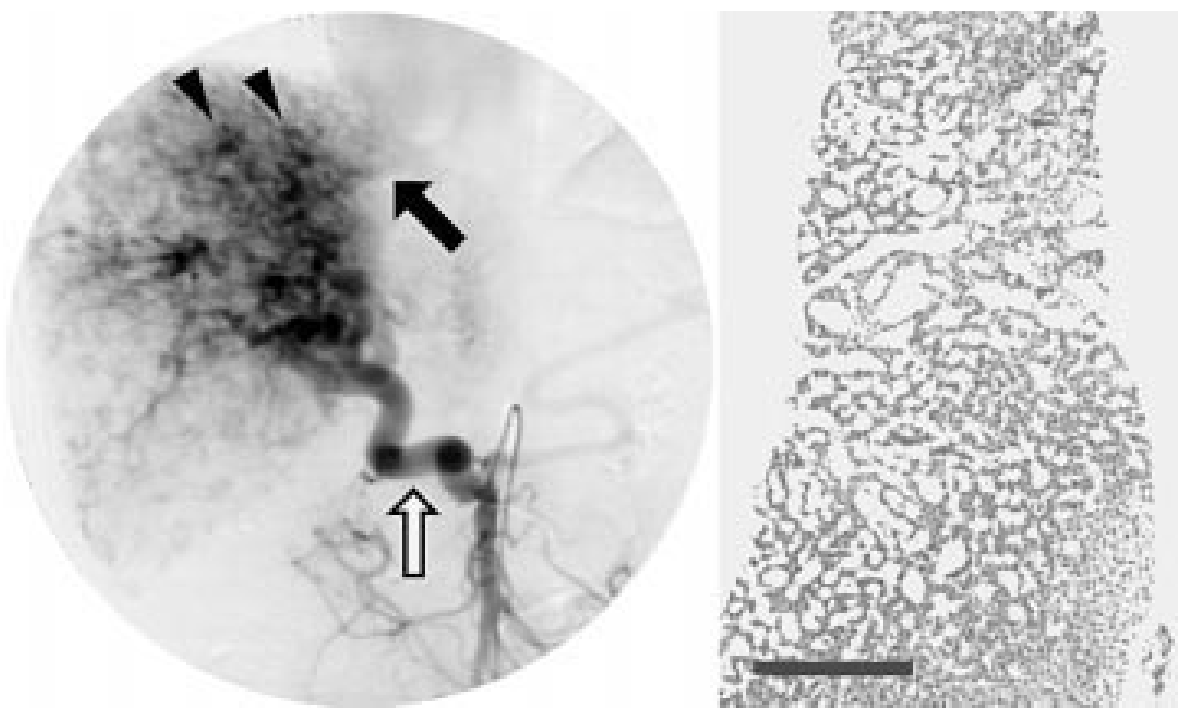

Figure 2 Left panel: Angiogram of the superior mesenteric artery. Note dilated feeding artery (white arrow), abundant dense mottled hepatogram (arrowheads), and early filling of hepatic vein (black arrow), which suggest multiple submacroscopic portal-systemic shunts in the liver. Right panel: Low power field micrograph of liver specimen obtained by needle biopsy. Haematoxylin-eosin stain. Note the diffusely dilated sinusoid in liver parenchyma. The bar indicates $0.5 \mathrm{~mm}$. 
that manganese exposure can result in extrapyramidal syndromes with signal changes on MRI, especially in the globus pallidus, where neuronal loss has been confirmed by pathological studies. ${ }^{910}$ Finally, this patient's parkinsonism was resistant to levodopa administration. A previous double blind study with levodopa failed to show improvement in manganese induced parkinsonism. ${ }^{11}$ From these findings we suggest that our patient has manganese induced parkinsonism caused by hereditary haemorrhagic telangiectasia with portal-systemic shunting.

Manganese delivery into the CNS is proposed to occur as follows. Ingested manganese in food is absorbed into the portal circulation as divalent manganese. Most absorbed manganese is excreted into bile, ${ }^{12}$ but a small portion enters the systemic circulation bound to transferrin. The manganesetransferrin complex will cross the blood-brain barrier by receptor mediated endocytosis into brain endothelial cells. Neurones in the nucleus accumbens and caudate-putamen have abundant transferrin receptors and preferentially take up the manganese-transferrin complex into the cell body. ${ }^{13}$ In neurones, manganese is delivered by axonal flow to nerve terminals in the ventral pallidum, globus pallidus, and substantia nigra, where manganese neurotoxicity occurs. ${ }^{14}$

As mentioned in our introduction, excessive portal-systemic shunting will cause hepatic encephalopathy, which was not observed in this patient. We therefore hypothesise that the portal-systemic shunting in our patient was compensated for by adequate liver function, but was too great to maintain the serum manganese level within normal limits. The normal level of serum ammonia and the mild impairment of indocyanine green disappearance with a raised manganese concentration support this hypothesis. Chronic iron deficiency might have promoted the uptake of manganese into the brain, because the anaemia must have caused deficiency of iron-transferrin complex, which has a fivefold greater affinity for the transferrin receptor than manganese-transferrin complex, and potentially inhibits manganese accumulation in the CNS. ${ }^{15}$

\section{Conclusions}

We report the case of a woman with hereditary haemorrhagic telangiectasia who showed manganese induced parkinsonism. This may be a new type of neurological complication in such patients. Levodopa resistant parkinsonism with telangiectasia suggests hereditary haemorrhagic telangiectasia, and a series of investigations should be undertaken to rule out a latent portal-systemic shunt.

\section{Authors' affiliations}

K Yoshikawa, M Matsumoto, M Hamanaka, M Nakagawa, Research Institute for Neurological Diseases and Geriatrics, Kyoto Prefectural University of Medicine, 465 Kawaramachi-Hirokoji, Kamigyo-ku, Kyoto 602-8566, Japan

Correspondence to: Dr Kenji Yoshikawa; kyoshika@koto.kpu-m.ac.jp

Received 20 February 2003

In revised form 11 March 2003

Accepted 21 March 2003

\section{REFERENCES}

1 Rendu H. Epistaxis répétées chez un sujet porteur de petits angiomes cutanés et muqueux. Gaz Hop (Paris) 1896;135:333-7.

2 Vincent $\mathbf{P}$, Plauchu $\mathrm{H}$, Hazan J, et al. A third locus for hereditary haemorrhagic telangiectasia maps to chromosome 12q. Hum Mol Genet 1995:4:45-9.

3 Románi G, Fisher M, Perl DP, et al. Neurological manifestations of hereditary telangiectasia (Rendu-Osler-Weber disease): report of 2 cases and review of the literature. Ann Neurol 1978;4:130-44.

4 Yekhlef F, Ballan G, Macia F, et al. Routine MRI for the differential diagnosis of Parkinson's disease, MSA, PSP, and CBD. J Neural Transm 2003;110:151-69

5 Tohgi H, Takahashi S, Abe T, et al. Symptomatic characteristics of parkinsonism and the width of substantia nigra pars compacta on MRI according to ischemic changes in the putamen and cerebral white matter: implications for the diagnosis of vascular parkinsonism. Eur Neurol 2001;46:1-10

6 Fahn S, Elton RL. Unified Parkinson's disease rating scale. In: Fahn S, Marsden CD, Goldstein M, et al, eds. Recent developments in Parkinson's disease, vol 2. Florham Park, NJ: MacMillan Healthcare Information, 1987: 153-63.

7 Halpern M, Turner AF, Citron BP. Hereditary hemorrhagic telangiectasia. An angiographic study of abdominal visceral angiodysplasias associated with gastrointestinal hemorrhage. Radiology 1968:90:1143-9.

8 Ejima A, Imamura T, Nakamura S, et al. Manganese intoxication during total parenteral nutrition. Lancet 1992;339:426.

9 Pal PK, Samii A, Calne DB. Manganese neurotoxicity: a review of clinical features, imaging and pathology. Neurotoxicology 1999:20:227-38

10 Jog MS, Lang AE. Chronic acquired hepatocerebral degeneration: case reports and new insights. Mov Disord 1995;10:714-22.

11 Lu C-S, Huang C-C, Chu N-S, et al. Levodopa failure in chronic manganism. Neurology 1994;44:1600-2.

12 Papavasiliou PS, Miller ST, Gotzias GC. Role of liver in regulating distribution and excretion of manganese. Am J Physiol 1966;41:21 1-16.

13 Aschner M, Aschner JL. Manganese neurotoxicity: cellular effects and blood-brain barrier transport. Neurosci Biobehav Rev 1991;15:333-40.

14 Eriksson H, Tedroff J, Thuomas KA, et al. Manganese induced brain lesions in Macaca fascicularis as revealed by positron emission tomography and magnetic resonance imaging. Arch Toxicol 1992;66:403-7

15 Karin M, Mintz B. Receptor-mediated endocytosis of transferring in developmental totipetent mouse teratocarcinoma stem cells. J Biol Chem 1981;256:3245-52. 